PSI-PR-96-35

October 1996

\title{
Annihilation into Channels with Strangeness and the OZI Rule Violationg
}

\author{
V.E. Markushin \\ Paul Scherrer Institute, 5232 Villigen PSI, Switzerland
}

\begin{abstract}
Two-step mechanisms in the $N \bar{N}$ annihilation and their role in the OZI rule violating reactions are discussed. In particular the two meson rescattering mechanism for $\pi \phi$ channel including all off-shell effects is typically two orders of magnitude bigger than the OZI tree level expectation and explains the observed ratio $\phi \pi / \omega \pi$ in the annihilation at rest. The rates for the final states including photons, $\gamma \omega$ and $\gamma \phi$, can be explained in the vector dominance model. The observed rate for $p \bar{p} \rightarrow \gamma \omega$ is suppressed due to destructive interference between the intermediate $\rho$ and $\omega$ states while the interference in $p \bar{p} \rightarrow \gamma \phi$ is required to be constructive leading to a large ratio $\gamma \phi / \gamma \omega$.
\end{abstract}

\section{Introduction}

In the nucleon-antinucleon annihilation near threshold, the channels with strangeness contribute only a few percent [1], nevertheless, the corresponding reactions are of big importance because they provide new information on reaction mechanisms, proton structure, and exotic states. Of special interest are the processes resulting in the violation of the Okubo-Zweig-Iizuka (OZI) rule [2, 3, 团. The recent experiments at LEAR have been very successful in providing the data on this subject [5, 6, 7, 8, 9, 10, 11, 12, 13, 14, 15, 16, 17, 18. A characteristic feature of the low energy $p \bar{p}$ annihilation is an abundant production of the $\phi$ mesons in some channels $(\phi \pi, \phi \gamma, \phi \rho, \phi \pi \pi, \phi \omega, \phi \phi)$ which is expected to be OZI suppresses on the tree level because the $\phi$ is dominantly a $\bar{s} s$ configuration.

\footnotetext{
${ }^{1}$ Invited contribution at LEAP-96, August 1996, Dinkelsbühl, Germany (to be published in Nuclear Physics B Proceedings Supplement).
} 
Because the OZI rule can be dynamically broken, it is important to understand whether the effects observed in the $p \bar{p}$ annihilation can result from known mechanisms of the OZI violation.

Two-step processes with ordinary hadrons as intermediate doorway states have been considered long ago and were shown to be important already in unitarity approximation (see [19 and references therein). In the case of $N \bar{N}$ annihilation two meson doorway mechanisms have been studied for various final states containing $\phi$ mesons in $p \bar{p}$ and $p \bar{d}$ reactions which violate the OZI rule [20, 21, 22, 23, 24, 25, 26, 27]. Another way of violating the OZI rule is production of flavor mixed or exotic (glueballs, hybrids) intermediate states, an approach which will not be discussed here. Furthermore, one can link the OZI rule violation to the nucleon structure by introducing an intrinsic $\bar{s} s$ component as in [28] (see also [29] and references therein for further details).

The goal of this paper is to review recent theoretical results concerning the evaluation of conventional two step mechanisms for $p \bar{p} \rightarrow \phi X$ as an explanation of the OZI rule violation. 'New physics' should be introduced only if these explanations fail. For earlier reviews we refer to [30, 31].

\section{The OZI Rule and Flavor Mixing}

The OZI rule [2, 3, 4] states that processes with disconnected quark lines in initial or final states are suppressed. For the heavy quarks $(c, b, t)$ this suppression is a consequence of the asymptotic freedom of QCD because the coupling constant $\alpha_{s}\left(q^{2}\right)$ becomes small, therefore the creation of a quark-antiquark pair is a perturbative process. The case of strange quarks belongs to the non-perturbative domain and involves more complicated dynamics.

One reason for the OZI rule violation is the flavor mixing in ordinary (nonexotic) hadrons. In QCD with massless $u, d, s$ quarks the hadrons would form $S U(3)$ multiplets, with the OZI suppression mechanism being trivially avoided for the states which are a superposition of all possible configurations. In reality the $s$ quark is significantly heavier than the $u$ and $d$ quarks, and the $S U(3)$ symmetry is broken. In particular, the physical states $\omega$ and $\phi$ are the superpositions of the $S U(3)$ singlet $\omega_{1}$ and octet $\omega_{8}$ states [2, 32]:

$$
\begin{aligned}
\left(\begin{array}{l}
\phi \\
\omega
\end{array}\right) & =\left(\begin{array}{cc}
\cos \Theta & -\sin \Theta \\
\sin \Theta & \cos \Theta
\end{array}\right)\left(\begin{array}{l}
\omega_{8} \\
\omega_{1}
\end{array}\right) \\
\omega_{1} & =\frac{u \bar{u}+d \bar{d}+s \bar{s}}{\sqrt{3}}=\sqrt{\frac{2}{3}} q \bar{q}+\sqrt{\frac{1}{3}} s \bar{s} \\
\omega_{8} & =\sqrt{\frac{1}{3}} q \bar{q}-\sqrt{\frac{2}{3}} s \bar{s}
\end{aligned}
$$

where $q \bar{q}=(u \bar{u}+d \bar{d}) / \sqrt{2}$. The so called ideal mixing corresponds to a complete 
decoupling of the $\bar{s} s$ and $\bar{q} q$ components:

$$
\Theta_{i}=\arctan \sqrt{1 / 2}=35.3^{\circ} \Longrightarrow \begin{aligned}
& \phi=s \bar{s} \\
& \omega=q \bar{q}
\end{aligned}
$$

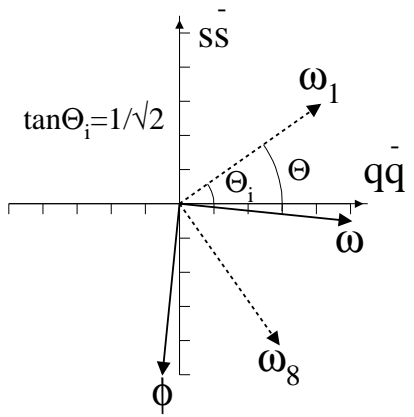

The Gell-Mann-Okubo mass formula with octet-singlet mixing gives the mixing angle $\Theta \approx 39^{\circ}$ [33]. The mixing angle can also be obtained from the partial widths of the $\omega$ and $\phi$ decays into $e^{+} e^{-}$

$$
\frac{\Gamma_{\omega \rightarrow e^{+} e^{-}}}{M_{\omega}}: \frac{\Gamma_{\phi \rightarrow e^{+} e^{-}}}{M_{\phi}}=\tan ^{2} \Theta
$$

leading to $\Theta=37.1^{\circ}$.

Since $\Theta \neq \Theta_{i}$ the OZI forbidden processes involving the $\phi$ production can go via the $s \bar{s}-q \bar{q}$ mixing. The corresponding amplitudes are expected to be proportional to the deviation from the ideal mixing $\left(\Theta-\Theta_{i}\right)^{2} \sim(1-4.2) \cdot 10^{-3}$ for $\Theta-\Theta_{i} \approx 1.8^{\circ}-3.7^{\circ}$. The OZI rule violation on the level of the flavor mixing is usually called nondramatic. Compared to the level given by the $\omega-\phi$ mixing, the low energy $p \bar{p}$ annihilation into the $\gamma \phi, \pi \phi$, and $\phi \phi$ channels appears to be rather dramatic exceeding it by 1-2 orders of magnitude. This problem will be discussed in detail in the following sections.

\section{The OZI Rule Violation in the $p \bar{p}$ Annihilation into Two Vector Mesons}

Table 1 shows the summary of the experimental data on the $p \bar{p} \rightarrow 1^{-}+1^{-}$ reactions at rest (the annihilation in liquid target predominantly occurs in the $S$-wave, in gaseous targets both the $S$ and $P$ waves contribute; the LX trigger in gas mainly corresponds to the $P$-wave annihilation). 
Table 1: The experimental branching ratios for $p \bar{p}$ annihilation at rest into $\rho \omega$, $\omega \omega, \gamma \omega, \rho \phi, \omega \phi, \gamma \phi$, channels.

\begin{tabular}{|c|c|c|c|}
\hline Reaction & BR & Condition & Ref. \\
\hline \multirow[t]{4}{*}{$\overline{p \bar{p} \rightarrow \rho \omega}$} & $5.4(6) \cdot 10^{-2}$ & gas. & |6] \\
\hline & $3.0(7) \cdot 10^{-2}$ & $\mathrm{~S}$ & 6 \\
\hline & $6.4(11) \cdot 10^{-2}$ & $\mathrm{P}$ & |6] \\
\hline & $2.3(2) \cdot 10^{-2}$ & liq. & 34 \\
\hline \multirow[t]{2}{*}{$p \bar{p} \rightarrow \omega \omega$} & $3.32(34) \cdot 10^{-2}$ & liq. & 8 \\
\hline & $1.4(6) \cdot 10^{-2}$ & liq. & 36 \\
\hline$p \bar{p} \rightarrow \gamma \omega$ & $6.8(18) \cdot 10^{-5}$ & liq. & [7] \\
\hline \multirow[t]{4}{*}{$\overline{p \bar{p} \rightarrow \rho \phi}$} & $3.4(8) \cdot 10^{-4}$ & gas. & [5] \\
\hline & $4.4(12) \cdot 10^{-4}$ & gas./LX & |5 \\
\hline & $3.4(10) \cdot 10^{-4}$ & ${ }^{1} S_{0}$ & 5 \\
\hline & $3.7(9) \cdot 10^{-4}$ & ${ }^{3} P_{J}$ & 5 \\
\hline \multirow[t]{5}{*}{$p \bar{p} \rightarrow \omega \phi$} & $6.3(23) \cdot 10^{-4}$ & liq. & 35 \\
\hline & $3.0(11) \cdot 10^{-4}$ & gas. & [5] \\
\hline & $4.2(14) \cdot 10^{-4}$ & gas./LX & 5 \\
\hline & $5.3(22) \cdot 10^{-4}$ & ${ }^{1} S_{0}$ & 5 \\
\hline & $2.9(14) \cdot 10^{-4}$ & ${ }^{3} P_{J}$ & 5 \\
\hline$p \bar{p} \rightarrow \gamma \phi$ & $1.7(4) \cdot 10^{-5}$ & liq. & 11 \\
\hline
\end{tabular}



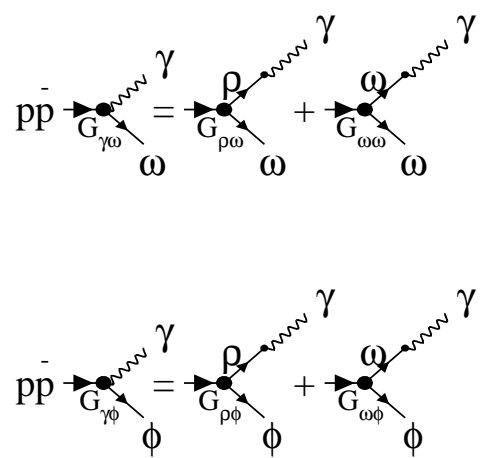

Figure 1: The amplitudes of the reactions $p \bar{p} \rightarrow \gamma \omega$ and $p \bar{p} \rightarrow \gamma \phi$ in the vector dominance model.

The vector dominance model (VDM) can be used to connect the amplitude of the reaction $p \bar{p} \rightarrow \gamma X$ with the amplitudes for $p \bar{p} \rightarrow \rho X$ and $p \bar{p} \rightarrow \omega X$ [37, 21, 7, \&]. The final states of interest for the OZI rule violation correspond to $X=\phi, \omega$.

The VDM relation has the form (see Fig. 1)

$$
\begin{aligned}
G_{\gamma \omega} & =\frac{\gamma_{\rho \gamma}}{m_{\rho}^{2}} G_{\rho \omega} F_{\rho \omega}+\frac{\gamma_{\omega \gamma}}{m_{\omega}^{2}} G_{\omega \omega} F_{\omega \omega} \\
G_{\gamma \phi} & =\frac{\gamma_{\rho \gamma}}{m_{\rho}^{2}} G_{\rho \phi} F_{\rho \phi}+\frac{\gamma_{\omega \gamma}}{m_{\omega}^{2}} G_{\omega \phi} F_{\omega \phi}
\end{aligned}
$$

where the amplitudes $G_{a b}$ correspond to the final states $a b$ and the coupling constants $\gamma_{\gamma \rho}$ and $\gamma_{\gamma \omega}$ are related by

$$
\gamma_{\gamma \rho}=3 \gamma_{\omega \rho}=m_{\rho}^{2} \frac{e}{f_{\rho}}=0.054 m_{\rho}^{2}
$$

The form-factors $F_{a b}\left(p_{a}^{2}\right)$ describe the off-shell behavior of the $p \bar{p} \rightarrow a b$ vertex, they are normalized by the condition $F_{\omega \omega}\left(m_{\omega}^{2}\right)=F_{\rho \omega}\left(m_{\rho}^{2}\right)=F_{\omega \phi}\left(m_{\omega}^{2}\right)=F_{\rho \phi}\left(m_{\rho}^{2}\right)=1$.

Since the final states have well defined $C$-parity $C=+1$, the allowed initial $p \bar{p}$ states are ${ }^{1} S_{0}$ and ${ }^{3} P_{J}$. Our analysis will be focused on the $S$-wave annihilation, therefore only the ${ }^{1} S_{0}$ state contributes. The corresponding partial width is

$$
\Gamma_{a b}=\frac{G_{a b}^{2} P_{a b}^{3}}{4 \pi}
$$

where $P_{a b}$ is the CMS momentum of the particles $a$ and $b$ in the final state.

From (6 9) and neglecting the mass difference of the $\omega$ and $\rho$ mesons the following result for the branching ratios is derived [37]

$$
\frac{B R(\gamma \omega)}{B R(\rho \omega)}=
$$




$$
\begin{aligned}
& =\left|F_{\rho \omega}+\frac{\sqrt{2}}{3} \sqrt{\frac{B R(\omega \omega)}{B R(\rho \omega)}} F_{\omega \omega} e^{i \beta_{\omega}}\right|^{2}\left(\frac{e}{f_{\rho}}\right)^{2}\left(\frac{P_{\gamma \omega}}{P_{\rho \omega}}\right)^{3} \\
& \frac{B R(\gamma \phi)}{B R(\rho \phi)}= \\
& =\left|F_{\rho \phi}+\frac{1}{3} \sqrt{\frac{B R(\omega \phi)}{B R(\rho \phi)}} F_{\omega \phi} e^{i \beta_{\phi}}\right|^{2}\left(\frac{e}{f_{\rho}}\right)^{2}\left(\frac{P_{\gamma \phi}}{P_{\rho \phi}}\right)^{3}
\end{aligned}
$$

where $\beta_{X}$ is the relative phase between the two terms corresponding to $\rho X$ and $\omega X$ intermediate states $(X=\omega, \phi)$.

\subsection{Reaction $p \bar{p} \rightarrow \gamma \omega$}

Using the experimental branching ratios $B R(\gamma \omega) / B R(\rho \omega)=(2.3 \pm 1.1) \cdot 10^{-3}$ and $B R(\omega \omega) / B R(\rho \omega)=1.1 \pm 0.3$ [6, 7, 8] one gets

$$
\left|F_{\rho \omega}+(0.49 \pm 0.07) F_{\omega \omega} e^{i \beta_{\omega}}\right|=0.49 \pm 0.13
$$

If $F_{\rho \omega} \approx F_{\omega \omega} \approx 1$, then $\beta_{\omega} \approx \pi$, i.e. there is a destructive interference between the $\rho$ and $\omega$ intermediate states with isospin $I=1,0$ in the total amplitude of the reaction $p \bar{p} \rightarrow \gamma \omega$. The result of the analysis by the Crystal Barrel Collaboration [7] obtained with a model formfactor [38] is $\cos \beta_{\omega}=-0.60 \pm 0.18$.

\subsection{Reaction $p \bar{p} \rightarrow \gamma \phi$}

Using the experimental branching ratios $B R(\gamma \phi) / B R(\rho \phi)=0.05 \pm 0.025$ and $B R(\omega \phi) / B R(\rho \phi)=1.6 \pm 1.1$ [5, 11] one gets

$$
\left|F_{\rho \phi}+(0.4 \pm 0.1) F_{\omega \phi} e^{i \beta_{\phi}}\right|=1.1 \pm 0.3
$$

If $F_{\rho \phi} \approx F_{\omega \phi} \approx 1$, then the data can be explained by a constructive interference between the $\rho$ and $\omega$ terms. The effect of the formfactor in this case can be more significant than for the $\gamma \omega$ final state because of a larger difference in the final momenta: $P_{\rho \phi}=0.28 \mathrm{GeV} / \mathrm{c}$ and $P_{\gamma \phi}=0.66 \mathrm{GeV} / \mathrm{c}$ (compare with the case $p \bar{p} \rightarrow \rho \omega, \gamma \omega: P_{\rho \omega}=0.53 \mathrm{GeV} / \mathrm{c}$ and $\left.P_{\gamma \omega}=0.78 \mathrm{GeV} / \mathrm{c}\right)$.

The analysis done in [8] arrived at the conclusion that the VDM prediction is too small to describe the experimental data. This conclusion, however, strongly depends on the model formfactor [38]. If we use the following formfactor

$$
F_{\rho \phi}\left(p_{\rho}^{2}\right)=\frac{\Lambda^{2}+P_{\rho \phi}^{2}}{\Lambda^{2}+P^{2}\left(p_{\rho}^{2}\right)}
$$

where $P\left(p_{\rho}^{2}\right)$ is the CMS momentum of the particles in the final state, and a similar one for the $\omega \phi$ vertex, than for $\Lambda=1 \mathrm{GeV} / \mathrm{c}$ the suppression factor is $F_{\rho \phi}(0)=0.75$ and the VDM relation (13) holds true for $\cos \beta_{\phi} \approx 1$. 
Thus the relative phases of the intermediate terms $I=0,1$ for the two reactions $p \bar{p} \rightarrow \gamma \omega, \gamma \phi$ are different, and this agrees with the observation that $\omega-\phi$ mixing cannot be the dominant mechanism of the $\phi$ production. Indeed, the OZI rule violation in the $\phi \omega$ and $\phi \rho$ channels, given by the ratios $B R(\phi \omega) / B R(\omega \omega) \sim B R(\phi \rho) / B R(\omega \rho) \sim 10^{-2}$, seems to exceed the level expected from the deviation from the ideal mixing. $2\left(\Theta-\Theta_{i}\right)^{2} \sim 0.4 \cdot 10^{-2}$, but is not dramatic. Thus a seemingly drastic violation of the OZI rule observed in the ratio $B R(\gamma \phi) / B R(\gamma \omega)=0.24 \pm 0.09$ [7, 11, 12] results from partial cancellation of the two terms $I=0,1$ for the $\gamma \omega$ channel and the constructive interference between the intermediate states for the $\gamma \phi$ channel. Therefore the core problem lies in the explanation of the $p \bar{p} \rightarrow \rho \phi$ and $p \bar{p} \rightarrow \omega \phi$ reactions which will be discussed in Sec. 7 .

\section{Two-Step Mechanism as Dynamical Breaking of the OZI Rule}

Reactions which are OZI forbidden in a tree approximation can proceed via twostep mechanisms without a violation of the OZI rule at the individual steps [19, 39, 40, 41]. Before discussing this mechanism in the $p \bar{p}$ annihilation we briefly illuminate it by the example of the $\phi \rightarrow \rho \pi$ decay.

\subsection{The OZI rule violation $\phi \rightarrow \rho \pi$ decay}

The width of the $\phi \rightarrow \rho \pi$ decay due to the $\omega-\phi$ mixing is given by the estimate

$$
\Gamma_{\phi \rightarrow \omega \rightarrow \rho \pi} \approx\left(\Theta-\Theta_{i}\right)^{2} \frac{g_{\omega \rho \pi}^{2} P_{\phi \rightarrow \rho \pi}^{3}}{12 \pi} \approx 0.16 \mathrm{MeV}
$$

where $g_{\omega \rho \pi}^{2} / 4 \pi=20.6 \mathrm{GeV}^{-2}$ 42 and $P_{\phi \rightarrow \rho \pi}$ is the CMS momentum of the particles in the final state. This is significantly smaller than the experimental value $\Gamma_{\phi \rightarrow \rho \pi}=0.57 \mathrm{MeV}$. The latter can be explained by the two step mechanism $\phi \rightarrow K \bar{K} \rightarrow \rho \pi$ 45, 46].

\footnotetext{
${ }^{2}$ The estimation can vary if the final state phase space factor is taken into account.
} 


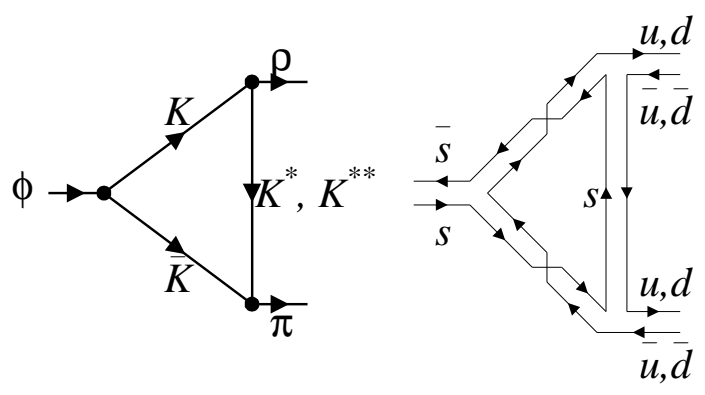

All the vertices are OZI allowed in this two step process. A simple estimate of the amplitude can be obtained in the unitarity approximation when the intermediate particles $K \bar{K}$ are on the mass shell. It gives the imaginary part of the amplitude which is of the right order of magnitude, but for a quantitative agreement the real part is essential, and the full calculation of the hadronic loops is needed [45, 46].

\subsection{Lipkin cancellations in hadronic loops}

Since two-step (one-loop) mechanisms tend to be large, one faces the problem of avoiding too large corrections to the OZI rule [19, 39, 40, 41, 43, 44]. It turns out that cancellations between various intermediate states can suppress the two step corrections in some cases.

The effect of cancellations for the mass operator in the meson nonets is demonstrated in Table 2 showing the relative sign of the various kaonic intermediate states contributing to the real part of the mixing amplitude in different $J^{P C}$ channels [44]. Nearly perfect cancellation between the $K \bar{K}, K^{*} \bar{K}, K \bar{K}^{*}, K^{*} \bar{K}^{*}$ terms explains a small deviation from the ideal mixing for $J^{P C}=1^{--}, 2^{++}, 3^{--}$mesons, while no cancellation occurs in the scalar sector where the mixing is known to be large.

A special case is the mass region above one and only one OZI allowed channel threshold. In this case the corresponding loop has an imaginary part which cannot be canceled by other terms. Therefore, with a sufficiently strong coupling to a single OZI allowed channel, dynamical breaking of the OZI rule can occur. The decay $\phi \rightarrow \rho \pi$ is a particular example of this effect. In the following section we consider how the two-step mechanism works in the case of $p \bar{p}$ annihilation.

\section{Two Meson Doorway Rescattering Mechanism in $p \bar{p} \rightarrow \phi \pi$}

The OZI rule violation in the $p \bar{p} \rightarrow \phi \pi$ at rest is rather strong: $B R(p \bar{p} \rightarrow$ $\phi \pi) / B R(p \bar{p} \rightarrow \omega \pi)=0.096 \pm 0.015$ [8, 11, 12]. The two-step mechanisms in 
Table 2: The relative sign of various intermediate states in the hadronic loop contributing to the $q \bar{q}-s \bar{s}$ mixing [44] and the corresponding deviation from the ideal mixing $\left(\Theta-\Theta_{i}\right)(\mathrm{deg})$.

\begin{tabular}{|c|cccc|c|}
\hline$J^{P C}$ & $K K$ & $K K^{*}$ & $K^{*} K$ & $K^{*} K^{*}$ & $\Theta-\Theta_{i}$ \\
\hline $1^{--}$ & + & - & - & + & $0.7-3.4$ \\
$2^{++}$ & + & - & - & + & $7-9$ \\
$3^{--}$ & + & - & - & + & $6-7$ \\
\hline $1^{+-}$ & 0 & - & - & + & $\sim 18$ \\
$1^{++}$ & 0 & - & - & + & $\sim 26$ \\
$0^{-+}$ & 0 & - & - & + & $45-58$ \\
$0^{++}$ & + & 0 & 0 & + & $\sim 36$ \\
\hline
\end{tabular}

$p \bar{p} \rightarrow \phi+X$ were studied in [21, 22, 23, 24, 25, 27]. The most important intermediate states are $K \bar{K}^{*}-K^{*} \bar{K}$ and $\rho \rho$, since they provide the maximum combined strength of the vertex functions (Fig.21). In the $\rho \rho$ case the strong annihilation step [47] (a hundred times bigger than the $\pi \phi$ branching ratio) is followed by the strong $\rho \pi \pi$ vertex and the $\phi \rho \pi$ vertex of moderate strength. In the $K \bar{K}^{*}$ case the annihilation step is a factor of 10 weaker [48], but it is followed by maximum strength for the remaining vertices in the second step.

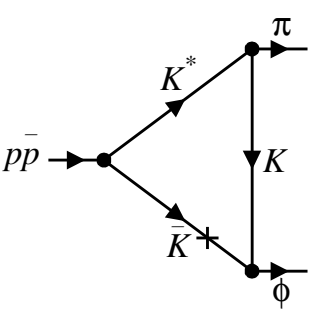

(a)

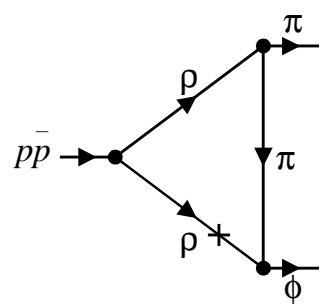

(b)

Figure 2: Two-step mechanisms in $p \bar{p} \rightarrow \phi+X$.

The scalar invariant amplitude has the form (see [27] for details)

$$
G(s)=\frac{i}{\pi} \int_{0}^{+\infty} \frac{K d K}{E_{1}(K)} \frac{A(K, s) F_{a}}{\left(E_{1 U}-E_{1}(K)+i \epsilon\right)},
$$


where $A(K, s)$ is the spectral density shown in Fig. 3 and $F_{a}$ is the form-factor describing the combined off-mass-shell effects for the intermediate state.
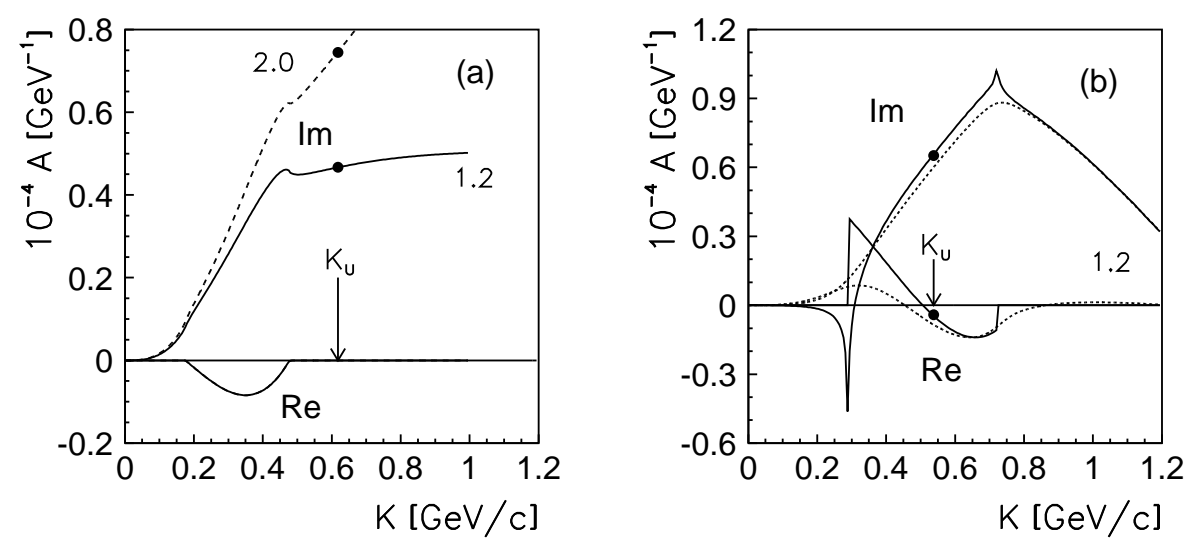

Figure 3: The spectral density $A(K, s)$ at threshold, $s=4 m_{p}^{2}$, vs. momentum $K$ : (a) the $K^{*} \bar{K}$-case, (b) the $\rho \rho$-case. The unitarity approximation amplitude $T_{U}=A\left(K_{U}, s\right)$ is marked by the full dots. The two curves for the imaginary part plotted in (a) correspond to different values of the form-factor parameter $\lambda_{b}$ (indicated in units of $\mathrm{GeV}$ ) describing the $t$-channel exchange in the second step [27]. The dotted lines in (b) correspond to a Breit-Wigner propagator for the $\rho$ meson.

The results of the one loop calculations are compared with the unitarity approximation and the experimental data in Table 3. The relative sign of the $K \bar{K}^{*}$ and $\rho \rho$ terms is not known. In the case of constructive interference the two step mechanism is in a good agreement with the data. Lipkin cancellations are not likely for this reaction [27].

Apart from annihilation at threshold the energy dependence carries potentially important information on the OZI rule violation. The energy dependence of the two step mechanism for $p \bar{p} \rightarrow \phi \pi$ has been estimated in [24]. The predictive power in this case suffers from too many unknown parameters. 
Table 3: Branching ratio for $p \bar{p}\left({ }^{3} S_{1}\right) \rightarrow \phi \pi^{0}$ in the unitarity approximation (UA) and with the off-mass shell effects taken into account (1-loop) in comparison with experiment [11. The form-factor parameters $\lambda_{a}$ and $\lambda_{b}$ [27] are in $\mathrm{GeV}$.

\begin{tabular}{|l|l|c|c|c|c|}
\hline \multicolumn{2}{|c|}{ Theory } & \multicolumn{4}{|c|}{ Two step mechanism } \\
\cline { 3 - 6 } \multicolumn{2}{|c|}{} & $K^{*} K$ & $\rho \rho$ & incoherent & maximum \\
\hline UA & $\lambda_{b}=\infty$ & $3.0 \times 10^{-4}$ & $2.0 \times 10^{-4}$ & $5.0 \times 10^{-4}$ & $9.9 \times 10^{-4}$ \\
& $\lambda_{b}=1.2$ & $0.63 \times 10^{-4}$ & $0.33 \times 10^{-4}$ & $0.96 \times 10^{-4}$ & $1.87 \times 10^{-4}$ \\
\hline 1-loop & $\lambda_{a}=1.5, \lambda_{b}=1.2$ & $0.55 \times 10^{-4}$ & $0.53 \times 10^{-4}$ & $1.09 \times 10^{-4}$ & $2.16 \times 10^{-4}$ \\
& $\lambda_{a}=2.0, \lambda_{b}=1.2$ & $0.69 \times 10^{-4}$ & $0.53 \times 10^{-4}$ & $1.22 \times 10^{-4}$ & $2.40 \times 10^{-4}$ \\
& $\lambda_{a}=2.0, \lambda_{b}=1.5$ & $1.59 \times 10^{-4}$ & $0.85 \times 10^{-4}$ & $2.44 \times 10^{-4}$ & $4.60 \times 10^{-4}$ \\
\hline \hline \multicolumn{5}{|c|}{ Crystal Barrel Collab. } & \multicolumn{5}{|c|}{$(5.5 \pm 0.7) \times 10^{-4}$} \\
\hline
\end{tabular}

\section{Two Meson Rescattering Mechanism in $p \bar{p} \rightarrow$ $\phi \phi$}

This reaction was studied in [20] in the unitarity approximation. The most important intermediate state is $K \bar{K}$ (Fig.(1a) for it has the maximum coupling to the final state. Notice that the $\phi \phi$ production via the $\omega-\phi$ mixing is strongly suppressed: $\sigma_{p \bar{p} \rightarrow \omega \omega \rightarrow \phi \phi} / \sigma_{p \bar{p} \rightarrow \omega \omega} \sim\left(\Theta-\Theta_{i}\right)^{4} \sim 10^{-5}$. The calculations of the two-step mechanism have correct magnitude in comparison with the experiment [15] as shown in Fig.4b. More data concerning the energy dependence of the $\phi \phi$ production would be very helpful for a further test of the mechanism considered.

(a)
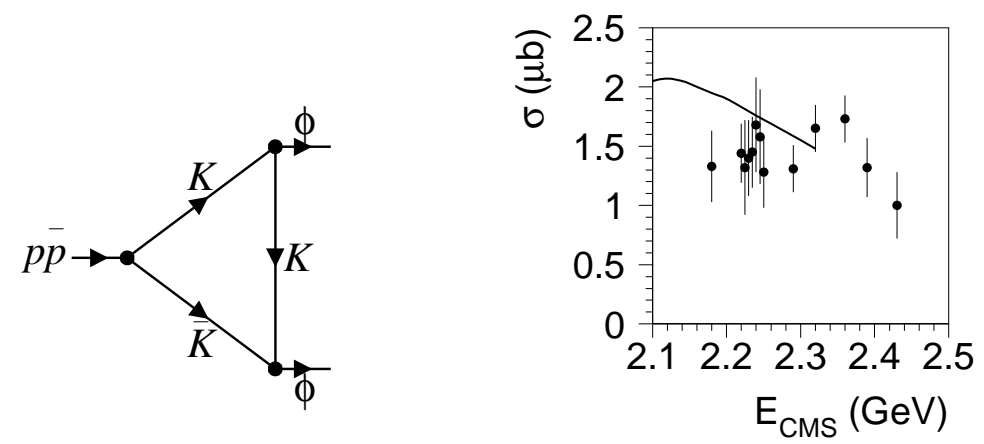

Figure 4: (a) The $K \bar{K}$ rescattering mechanism in $p \bar{p} \rightarrow \phi \phi$. (b) The energy dependence of the $p \bar{p} \rightarrow \phi \phi$ cross section calculated for the two step mechanism [20]. Experimental data from [15]. 


\section{The OZI Violation in $p \bar{p} \rightarrow \phi \pi^{+} \pi^{-}, \phi \rho, \phi \omega$}

Table 1 summarizes the experimental data on the OZI rule violation in the $\phi \pi^{+} \pi^{-}$ and $\phi \rho$ channels at different energies. The OZI rule violation in these channels is not dramatic compared with the level given by the $\omega-\phi$ mixing $\left(\Theta-\Theta_{i}\right)^{2} \sim$ $4 \cdot 10^{-3}$. The measured branching ratios for the reactions $p \bar{p} \rightarrow \phi \rho, \phi \omega$ at rest are given in Tab. 1. The OZI rule violation in the $\phi \omega$ channel, $B R(\phi \omega) / B R(\omega \omega)=$ $(19 \pm 7) \cdot 10^{-3}$ (liquid) [8, 35], is comparable with the effect in the $\phi \rho$ channel.

Table 4: The relative probabilities for the $\phi \rho, \omega \rho$ and $\phi \pi^{+} \pi^{-}, \omega \pi^{+} \pi^{-}$channels in the $p \bar{p}$ annihilation vs. antiproton momentum (in units $10^{-3}$ ).

\begin{tabular}{|c|c|c|c|}
\hline$p_{\bar{p}}(\mathrm{GeV} / \mathrm{c})$ & $\frac{B R(\phi \rho)}{B R(\omega \rho)}$ & $\frac{B R\left(\phi \pi^{+} \pi^{-}\right)}{B R\left(\omega \pi^{+} \pi^{-}\right)}$ & Ref. \\
\hline 0 (gas) & $6.3 \pm 1.6$ & & {$[5,6]$} \\
\hline $0($ gas/LX) & $7.5 \pm 2.4$ & & [5, 6] \\
\hline 0 (liq.) & & $7.0 \pm 1.4$ & 34 \\
\hline 0.76 & $9 \pm 5$ & $10.0 \pm 2.4$ & 49 \\
\hline 1.2 & & $11_{-4}^{+3}$ & 50 \\
\hline 2.3 & $22 \pm 13$ & $21 \pm 5$ & 51] \\
\hline 3.6 & & $9_{-7}^{+4}$ & 50 \\
\hline
\end{tabular}

The two-step mechanisms with two particles in the intermediate state were considered in the unitarity approximation in [23] where the calculated branching ratios for the $\phi \rho$ and $\phi \omega$ channels were found to be almost two orders of magnitude smaller than the data. The off-mass-shell corrections have not yet been calculated for these processes. It is also not excluded that the intermediate states with more than two particles (like $\rho \rho \pi, K^{*} \bar{K} \pi$ in the case of $\phi \rho$ ) are important.

\section{Conclusion}

Two step mechanisms play an important role in the observed violations of the OZI rule in the $p \bar{p}$ annihilation at low energies.

- The two meson rescattering mechanisms for $\bar{p} p \rightarrow \pi \phi$ including all off-massshell effects have a size almost two orders of magnitude bigger than the OZI tree level expectations, in agreement with the measured branching ratios at rest. The $K^{*} \bar{K}$ rescattering mechanism does not request any unexplained OZI violation for the individual vertices in the diagrams.

- The $\phi \phi$ production in the $p \bar{p}$ annihilation at $\sqrt{s}<2.5 \mathrm{GeV}$ can be explained by the two step mechanism with the intermediate state $K \bar{K}$.

- The $\phi \gamma / \omega \gamma$ ratio in the $p \bar{p}$ annihilation at rest can be explained by the VDM. The $(\rho \rightarrow \gamma)+(\omega \rightarrow \gamma)$ interference is destructive for $\omega \gamma$ and constructive for $\phi \gamma$. 
Thus the most drastic violations of the OZI rule can be explained by the known mechanism of rescattering. In particular no substantial $s \bar{s}$ component in the nucleon is required. Further studies of the OZI rule breaking due to two step corrections in other annihilation channels $(\phi \rho, \phi \pi \pi, \phi \omega)$ and the energy dependence of the $\phi$ production would be very desirable.

\section{Acknowledgements}

The author would like to thank M.P. Locher and S. von Rotz for fruitful collaboration and O. Gorchakov, F. Lev, and M. Sapozhnikov for useful discussions.

\section{References}

[1] Compilation of Cross Sections, III: $p$ and $\bar{p}$ induced reactions, CERN-HERA84-01, CERN, 1984.

[2] S. Okubo, Phys. Lett. 5 (1963) 165.

[3] G. Zweig, CERN Preprint 8419 / TH-412 (1964).

[4] I. Iizuka, Progr. Theor. Phys. Suppl. 37 (1966) 21.

[5] J. Reinfenröther, et al. Phys. Lett. B267 (1991) 299.

[6] P. Weidenauer, et al., Phys. Lett. B267 (1991) 299.

[7] C. Amsler et al., Phys. Lett. B311 (1993) 371.

[8] C. Amsler et al., Z. Phys. C58 (1993) 175.

[9] K. Braune, Nucl. Phys. A558 (1993) 269c.

[10] M.A. Faessler et al., Phys. At. Nucl. 57 (1994) 1693.

[11] C. Amsler et al., Phys. Lett. B346 (1995) 363.

[12] U. Wiedner, Proc. III Conf. on Low Energy Antiproton Physics, Bled, 1994, Eds. G. Kernel, P. Križan, M. Mikuž, World Sci., 1995, p. 319.

[13] V.G. Ableev et al., Phys. At. Nuclei 57 (1994) 1716.

[14] V.G. Ableev et al., Phys. Lett. B334 (1994) 237.

[15] M. Macri et al., . Proc. of the Second Biennial Conference on Low-Energy Antiproton Physics, Courmayeur, Italy, 1992, Eds. C. Guaraldo, F. Iazzi, A. Zenoni, North-Holland, Amsterdam, 1993, p.27c. 
[16] R.T. Jones, Proc. III Conf. on Low Energy Antiproton Physics, Bled, 1994, Eds. G. Kernel, P. Križan, M. Mikuž, World Sci., 1995, p. 326.

[17] U. Wiedner, Talk at LEAP-96, this volume.

[18] V. Lucherini, Talk at LEAP-96, this volume.

[19] H.J. Lipkin, Nucl. Phys. B244 (1984) 147.

[20] Y. Lu, B.S. Zou, and M.P. Locher, Z. Phys. A345 (1993) 207.

[21] M.P. Locher, Yang Lu, and B.S. Zou, Z. Phys. A347 (1994) 281.

[22] M.P. Locher and Yang Lu, Z. Phys. A351 (1994) 83.

[23] D. Buzatu and F.M. Lev, Phys. Lett. B329 (1994) 143.

[24] D. Buzatu and F.M. Lev, JINR preprint, E4-94-158, Dubna, 1994.

[25] D. Buzatu and F.M. Lev, Phys. Rev. C51 (1995) R2893.

[26] D. Buzatu and F.M. Lev, Phys. At. Nucl. 58 (1995) 480.

[27] O. Gortchakov, M.P. Locher, V.E. Markushin, and S. von Rotz, Z. Phys. A353 (1996) 447.

[28] J. Ellis, M. Karliner, D.E. Kharzeev, M.G. Sapozhnikov, Phys. Lett. B353 (1995) 319.

[29] T. Gutsche, G.D. Yen, A. Faessler, Talk at LEAP-96, this volume.

[30] M.P. Locher, Proc. III Conf. on Low Energy Antiproton Physics, Bled, 1994, Eds. G. Kernel, P. Križan, M. Mikuž, World Sci., 1995, p. 38.

[31] M.G. Sapozhnikov, Proc. III Conf. on Low Energy Antiproton Physics, Bled, 1994, Eds. G. Kernel, P. Križan, M. Mikuž, World Sci., 1995, p. 355.

[32] J. Arafune, M. Fukugita, and Y. Oyanagi, Phys. Lett. 70B (1977) 221.

[33] Review of Particle Properties, Phys. Rev. D54 (1996) 98.

[34] R. Bizzari et al., Nucl. Phys. B14 (1969) 169.

[35] R. Bizzari et al., Nucl. Phys. B27 (1971) 140.

[36] M. Bloch, G. Fontaine and E. Lillestol, Nucl. Phys. B23 (1970) 221.

[37] B. Delcourt, J. Layssac, and E. Pelaquier, Proc. of the Workshop on Physics at LEAR with Low Energy Antiprotons, Erice 1982, Plenum, New York, 1984 , p.305. 
[38] F. von Hippel and C. Quigg, Phys. Rev. D5 (1972) 624.

[39] H.J. Lipkin, Phys. Lett. B179 (1986) 278.

[40] H.J. Lipkin, Nucl. Phys. B291 (1987) 720.

[41] H.J. Lipkin, Phys. Lett. B225 (1989) 287.

[42] M. Gell-Mann, D. Sharp, and W. Wagner, Phys. Rev. Lett. 8 (1962) 261.

[43] P. Geiger and N. Isgur, Phys. Rev. Lett. 67 (1991) 1066.

[44] H.J. Lipkin and B.S. Zou, Phys. Rev. D53 (1996) 6693.

[45] J. Pasupathy, Phys. Rev. D12 (1975) 2929; J. Pasupathy and C.A. Smith, Phys. Lett. 61B (1976) 469.

[46] R. Tegen and J. Willrodt, Nuovo Cimento 43A (1978) 495.

[47] A. Cieply, M.P. Locher, and B.S. Zou, Z. Phys. A345 (1993) 41.

[48] B. Conforto et al., Nucl. Phys. B3 (1967) 469.

[49] A.M. Cooper et al., Nucl.Phys. B146 (1978) 1.

[50] R.A. Donald et al., Phys.Lett. B61 (1976) 210.

[51] C.K. Chen et al., Nucl.Phys. B130 (1977) 269. 